\title{
Sleep quality and duration in relation to memory in the elderly: Initial results from the Hellenic Longitudinal Investigation of Aging and Diet
}

\author{
A. Tsapanou ${ }^{\mathrm{a}, \mathrm{b}, *}, \mathrm{Y} \mathrm{Gu}^{\mathrm{b}}$, D.M. O’Shea ${ }^{\mathrm{c}}$, M. Yannakoulia ${ }^{\mathrm{d}}$, M. Kosmidis ${ }^{\mathrm{e}}$, E. Dardiotis ${ }^{\mathrm{f}}$, G. Hadjigeorgiou ${ }^{\mathrm{f}}$, \\ P. Sakka ${ }^{g}$, Y. Stern ${ }^{b}$, N. Scarmeas ${ }^{a, b}$ \\ a Department of Social Medicine, Psychiatry and Neurology, National and Kapodistrian University of Athens, Athens, Greece \\ ${ }^{\mathrm{b}}$ Cognitive Neuroscience Division, Department of Neurology and The Taub Institute for Research on Alzheimer's Disease and the Aging Brain, Columbia University Medical Center, \\ New York, NY, USA \\ c Department of Clinical and Health Psychology, University of Florida, FL, USA \\ ${ }^{\mathrm{d}}$ Department of Nutrition and Diabetics, Harokopio University, Athens, Greece \\ e Department of Psychology, Aristotle University of Thessaloniki, Greece \\ ${ }^{\mathrm{f}}$ Department of Neurology, Faculty of Medicine, University of Thessaly, Larissa, Greece \\ ${ }^{\mathrm{g}}$ Athens Association of Alzheimer's Disease and Related Disorders, Athens, Greece
}

\section{A R T I C L E I N F O}

Article history:

Received 23 December 2016

Revised 10 April 2017

Accepted 24 April 2017

Available online 26 April 2017

\section{Keywords:}

Sleep quality

Sleep duration

Memory

Elderly

\begin{abstract}
A B S T R A C T
Background: Sleep is crucial for cognition, particularly for memory, given its complex association with neurodegenerative processes. The aim of the present study was to examine the association between sleep quality as well as sleep duration and memory performance in a Greek elderly population.

Setting: Cross-sectional design in the Hellenic Longitudinal Investigation of Aging and Diet (HELIAD), a population representative study of Greek elderly (65 years or older).

Methods: Data from 1589 participants free of sleep medication were included. Sleep quality was estimated by using the Sleep Scale from the Medical Outcomes Study. An extensive neuropsychological assessment examining memory was administered to each participant. Linear regression analyses were used to examine whether sleep quality (higher score, poor quality) and/or sleep duration were associated with memory expressed in the form of a z-score. Age, sex, education, and body mass index were included as covariates. The main analyses were conducted first on the total sample, then with the exclusion of demented participants, and finally with the exclusion of both demented and participants with Mild Cognitive Impairment (MCI). We then conducted further analyses on the non-demented, non-MCI group, initially stratified by Apolipoprotein E-\&4 gene. We further examined the role of co-morbidities, as well as the association between sleep duration groups and memory. We also explored any interaction effect between sex and sleep quality/duration on memory. We then examined the associations between components of sleep measures and memory scores. Lastly, we examined the associations between sleep quality/duration and verbal/non-verbal memory separately.

Results: In the total sample, we noted significant associations between sleep duration and memory $(B=-0.001, p \leq 0.0001)$, but not for sleep quality and memory $(B=-0.038, p=0.121)$. After excluding the demented participants, the associations were significant for: sleep quality and memory $(B=-0.054, p=0.023)$, and sleep duration and memory $(B=-0.001, p \leq 0.0001)$. After excluding both the $\mathrm{MCI}$ and the demented subjects, the associations between sleep quality and memory $(B=-0.065$, $p=0.006)$, and sleep duration and memory $(B=-0.001, p=0.003)$ were still significant. The association between the sleep duration groups and memory function was also significant, such that poor memory performance was associated with the longer sleep duration group. The results remained significant even after controlling for the co-morbidities, as well as after adding in the model anxiety and depression as covariates. Associations between sleep quality and memory, and sleep duration and memory were present in the $A p o E-\varepsilon 4$ non-carriers. The individual sleep questions that were probably shown to be driving
\end{abstract}

Abbreviations: MCI, Mild Cognitive Impairment; AD, Alzheimer's Disease; MOS, Medical Outcomes Study; HELIAD, Hellenic Longitudinal Investigation of Aging and Diet.

* Corresponding author at: Cognitive Neuroscience Division, Department of Neurology, Columbia University College of Physicians and Surgeons, PH18-326, 622 West 168th St, New York, NY 10032, USA.

E-mail address: at2859@cumc.columbia.edu (A. Tsapanou). 
the associations between sleep and memory were: time to fall asleep, sleep not quiet, getting enough sleep to feel rested upon waking in the morning, and getting the amount of sleep needed. Sleep duration was associated with both verbal and non-verbal memory, while sleep quality was only associated with verbal memory.

Conclusion: Poor sleep quality and longer sleep duration were linked to low memory performance, independent of demographic and clinical factors, in a large sample of cognitively healthy older Greek adults. Other parameters than sleep and memory measurements could play an important role on the association. Levels of melatonin, or circadian rhythms dysregulation might play a crucial role in the above associations.

(C) 2017 Elsevier Inc. All rights reserved.

\section{Introduction}

Aging is associated with changes in sleep patterns. Self-reported sleep problems seem to reflect poor overall quality of sleep, which has also been linked to changes in cognition (Basner et al., 2007; Miyata et al., 2013; Ohayon, 2004; Ohayon et al., 2004; Ramos et al., 2013). Sleep quality has been associated with cognitive performance in the elderly (Gildner et al., 2014). Specifically, maintaining good sleep quality seems to promote better cognition and could protect against age-related cognitive decline (Scullin \& Bliwise, 2015).

Specifically regarding the association between sleep and memory, both sleep quality and sleep duration have been linked to memory performance (Miyata et al., 2013). According to a recent study, good sleep quality has been found to promote recognition memory (Monaghan et al., 2016). Poor sleep quality has been linked to a decline in declarative memory in another study (Backhaus et al., 2007). Other findings have shown a significant effect of both sleep quality and sleep duration on episodic memory (Aly \& Moscovitch, 2010). Sleep duration has also been linked to episodic memory in a separate study of older adults (Nebes et al., 2009).

Memory is a quite general and at the same time complex cognitive function including many different underlying cognitive processes such as verbal and non-verbal ones. Verbal memory has been linked to both sleep quality and sleep duration (Clemens, Fabo, \& Halasz, 2005; Mu et al., 2005). Literature examining the linkage between non-verbal memory and sleep is comparatively smaller, but associations mostly with sleep duration have been reported (Chee \& Chuah, 2007; Fernandez-Mendoza et al., 2010).

However, there are conflicting results on the associations between sleep and memory performance. According to a recent study, sleep quality, was not related to poor performance on memory or other cognitive tasks in older adults (Kawai et al., 2016). Moreover, a different study showed that there were no significant changes in memory of older adults in relation to sleep quality (Scullin, 2013). As for the sleep duration, the existing literature shows mixed results on the associations between short and long sleep durations and cognition (Faubel et al., 2009; Xu et al., 2011).

The existing literature on sleep and memory also account for the impact of Apolipoprotein E genotype (ApoE- $\varepsilon 4)$. ApoE- $\varepsilon 4$ has been associated with both sleep patterns (Gottlieb et al., 2004) and memory performance (Zlatar et al., 2016). However, within this literature there is also a lack of associations between ApoE$\varepsilon 4$ and risk of specific sleep problems (Larkin et al., 2006; Saarelainen et al., 1998; Thakre, Mamtani, \& Kulkarni, 2009; Tsapanou et al., 2015).

There are gaps in the existing literature examining the association between sleep and memory. First, many studies are based on small sample sizes (Aly \& Moscovitch, 2010; Miyata et al., 2013; Monaghan et al., 2016) or are focused on younger age groups, lim- iting its environmental validity (Kronholm et al., 2009; Monaghan et al., 2016). Furthermore, there is limited relevant literature in Mediterranean countries. In addition, existing research on this topic typically has not incorporated a comprehensive neurological evaluation which would allow for detailed differential diagnosis or an accurate description of the cognitive status of each participant. Furthermore, not many studies have focused on memory function. Another study examining sleep quality/quantity and cognitive function in the elderly, used computer-assisted interviews and only self-completion questionnaires for the evaluation of cognition and sleep, limiting the accuracy and the validity of the results (Miller et al., 2014). The same study was also focused on general cognitive function and not specifically on memory. Two other large population based studies which examined the association between sleep duration and cognitive function used either just only a brief assessment of cognitive status (a modified version of the Mini Mental State Examination [MMSE]) or a delayed word recall test (Faubel et al., 2009; Xu et al., 2011, 2014). Finally, most studies on this topic examine the influence of either sleep quality or sleep duration but do not examine the influence of both these variables on memory.

The present study was conducted in an attempt to bridge some of these gaps. More precisely, we aimed to examine the associations between self-reported sleep quality/duration and memory, in a large sample of older Greek adults; a population that is under-represented with regard to this topic. The data from this study may also permit future cross-cultural comparisons on this topic with other populations.

\section{Methods}

\subsection{Participants}

Participants were drawn from the Hellenic Longitudinal Investigation of Aging and Diet (HELIAD). HELIAD is a population-based, multidisciplinary, collaborative study designed to estimate the prevalence and incidence of Mild Cognitive Impairment ( $\mathrm{MCI}$ ), Alzheimer's Disease (AD), other types of dementia, as well as other neuropsychiatric conditions of aging in the Greek population. The study includes several demographic, medical, social, environmental, clinical, nutritional, and neuropsychological determinants as well as the lifestyle activities of each participant. HELIAD has been approved by the relevant Institutional Review Board. More detailed information about the study can be found in previously published work (Dardiotis et al., 2014).

\subsection{Sleep measures}

Sleep quality was assessed using the Sleep Scale from the Medical Outcomes Study (MOS). This scale is a self-reported 12-item questionnaire (Spritzer \& Hays, 2003). Based on the manual of 
the specific scale (Hays, Sherbourne, \& Mazel, 1995), in the current study, sleep quality was examined by the Sleep Index II, by averaging the sum of the following questions: During the past 4 weeks: 1. "How long did it usually take for you to fall asleep?" 2. "Feel that your sleep was not quiet (moving restlessly, feeling tense, speaking, etc., while sleeping)?" 3. "Get enough sleep to feel rested upon waking in the morning?" 4. "Awaken short of breath or with a headache?" 5. "Feel drowsy or sleepy during the day?" 6. "Have trouble falling asleep?" 7. "Awaken during your sleep time and have trouble falling asleep again?" 8. "Have trouble staying awake during the day?" 9. "Get the amount of sleep you needed?". Each of the questions has a possible rating of 1-6, based on the frequency of the sleep problem (see Appendix A). The sum of the sleep quality variable had a range of $1-54$. Based on the manual, we reversed the sleep items needed in order for a higher score to indicate greater sleep dysfunction.

In order to also examine sleep duration, we used the following question: "On the average, how many hours did you sleep each night during the past 4 weeks? Write in number of hours and minutes per night." The final variable used was the sum of the total duration calculated in minutes. To further examine any association between sleep duration ranges and memory performance, we differentiated three sleep duration groups. Large impact studies examining the association between sleep and memory have used as sleep duration cut-offs the following: $<6 \mathrm{~h}, 6-8 \mathrm{~h},>8 \mathrm{~h}$ (Kronholm et al., 2009; Miller et al., 2014; Xu et al., 2011). Based on those, we created a trichotomous sleep duration variable: the short-sleepers (<360 $\mathrm{min})$, the regular-sleepers (360-480 $\mathrm{min}$ ), and the long-sleepers ( $>480 \mathrm{~min}$ ).

For the purposes of our supplementary analyses, we used each individual sleep question of the sleep scale, with possible rating 16 and we reversed the items needed so that higher score indicates greater sleep dysfunction (see Appendix A).

Regarding reliability and validity of the MOS sleep scale in a Greek population, the following steps were taken: first, we followed a process of translation and back translation before finalizing the questionnaire form that was administered to our participants. Furthermore, as sleep quality (Sleep Index II) is concerned, in our group of non-demented, non MCI older ( $\geq 65 \mathrm{y} .0$.) participants $(\mathrm{N}=1338)$, we conducted reliability analyses which revealed a high internal consistency (Cronbach's $\alpha=0.729$ ). We further associated the sleep duration question from the MOS with the one from the Athens Physical Activity Questionnaire (APAQ) Kavouras et al., 2016 asking: During the last 7 days how many hours (on average) per day did you sleep (including siesta)? As for the sleep quality, we associated the MOS Sleep Index II with the equivalent answers from the Neuropsychiatric Inventory (NPI). Specifically, we computed a new continuous variable summing up the following sleep quality questions from the NPI: Changes observed during the past month in; "Difficulty falling asleep, Getting up during the night, Awakening too early in the morning, and Sleeping excessively during the day." Questions are dichotomous and the total amount of participants answered them was $\mathrm{N}=690$. In order to examine the associations between the two measures we used bivariate correlations. The Mean of the sleep duration from the APAQ was 7.2, with SD: 1.5. The correlation between the two sleep duration variables was statistically significant on a $p$ level $\leq 0.0001(r=0.716)$. The correlation between the two sleep quality variables was also statistically significant $(r=0.357, p \leq 0.0001)$. Thus, both sets of correlations support the evidence of convergent validity with regard to the MOS scale.

Last but not least, the existing literature shows a significant correlation between self-reported sleep problems and actual objective sleep measurements. Specifically, self-reported sleep duration has been correlated with objective polysomnographic characteristics (Armitage et al., 1997), and self-reported sleep quality with actig- raphy and polysomnography (Hita-Yanez, Atienza, \& Cantero, 2013; Landis et al., 2003), boosting the case that self-reported sleep questionnaires can reflect aspects of actual sleep.

\subsection{Clinical - cognitive evaluations}

A structured, in-person interview, consisting of a medical history report and physical examination, was conducted for each participant during each of the visits. All participants provided information about their current health status, any neurological condition, past medical problems, surgeries they might have been through, hospitalizations, and any use of medication. They also answered valid questionnaires about their daily activities, physical exercise, and diet. We used the Blessed Dementia Scale in order to examine any change in their self-care habits, activities, physical and cognitive function as well (Blessed, Tomlinson, \& Roth, 1968). In addition, the Lawton Instrumental Activities of Daily Living was used in order to examine the capacity of each participant to correspond independently to daily activities (Lawton \& Brody, 1969).

At the beginning of the evaluation each participant underwent a test for illiteracy which involved the participant reading a list of words and letters. In addition, a neuropsychological battery examining memory was administered. We examined verbal and nonverbal memory. To assess episodic verbal memory performance, we used the Greek Verbal Learning Test (GVLT). This is the Greek version of the California Verbal Learning Test, which assesses encoding, recall and recognition of 16 words which are initially read to the participant. For the purposes of the current study, for the evaluation of the verbal memory, we used an average composite of total raw scores counting the total sum of corrects (trials 1-5), immediate recall, and delayed recall (after a 30-min delay). Nonverbal memory was assessed by using the immediate and delayed recall scores from the Medical College of Georgia Complex Figure Test (GCFT). This task is a commonly used neuropsychological measure assessing visuospatial construction and non-verbal memory, which includes four alternate forms for repeating assessments. The participant is asked to copy a complex figure as accurate as possible. The examiner then removes the figure and the participant is asked to draw it. Following a delay of approximately $30 \mathrm{~min}$, the participant is asked to draw the figure again (Meador et al., 1993; Vlahou et al., 2013) For the total memory performance score, we used the sum of verbal and non-verbal tests (sum 5-trials correct, GVLT immediate/delayed recall, GCFT immediate/delayed recall).

First, z-scores were derived for each test, using the means and standard deviations (SD) calculated from the scores of the nondemented participants. The z-scores were calculated by subtracting the mean score from the individual score, and divided by the standard deviation. A higher z-score indicates better memory performance.

The diagnosis of the clinical/ cognitive status of each participant was reached through diagnostic consensus meetings of all the researchers and main investigators, both neurologists and neuropsychologists.

In an attempt to examine other sleep disorders that may be present in our sample, we collected information about; Restless Legs Syndrome (RLS) Allen et al., 2003; Garcia-Borreguero et al., 2006, sleep and nighttime behavior disorders from the Neuropsychiatric Inventory (NPI) Cummings et al., 1994, Rapid Eye Movements sleep Behavior Disorder (RBD) from the Semiqualified Clinical Fluctuating Cognition Rating Scale (Walker et al., 2000), as well as sleep duration (including siesta) from the APAQ (Kavouras et al., 2016).

\subsection{Covariates}

Age (years), education (years), and body mass index (BMI) were used as continuous variables. Sex was used as a dichotomous variable. 
Co-morbidities: In the present study, co-morbidities were recorded by using a 23-item-clinical conditions- questionnaire. Specifically, information was collected for: hypertension, diabetes mellitus, coronary disease, myocardial infraction, congestive heart failure, arrhythmia, any other heart disease, dyslipidemia, COPD or other pulmonary disease, thyroid disease, hyperthyroidism, hypothyroidism, liver disease, renal insufficiency, peptic ulcer disease, cancer, arthritis, illicit drug use, traumatic brain injury (with loss of consciousness), seizure, syphilis, Parkinson's disease, Huntington's disease, multiple sclerosis, B-12 deficiency, normal pressure hydrocephalus, down syndrome, any other medical condition or operation. We created a continuous variable summing up the total number of clinical co-morbidities of each participant.

Based on the existing literature suggesting specific clinical factors being correlated with sleep problems and cognition (Riegel \& Weaver, 2009; Yaffe et al., 2011; Young, Skatrud, \& Peppard, 2004), we further created a shorter variable including the sum of the 6 following parameters: hypertension, diabetes mellitus, coronary artery disease, myocardial infarction, congestive heart failure, and stroke. We then examined the association between sleep quality/duration and memory performance adding this new variable as a covariate.

As there is evidence that depression can be related to cognitive and specifically memory impairment (Grewe et al., 2016; Jorm, 2000; Schmutte et al., 2007), we also included it as a covariate on a secondary analysis. The specific variable was drawn from the Geriatric Depression Scale (GDS), which is a 15 -item selfreported questionnaire. In the analysis, GDS was used as a dichotomous variable, based on the existing literature (Fountoulakis et al., 1999; Yesavage, 1988). Anxiety was drawn from the Hospital anxiety and depression scale. This is a 7-item self-reported questionnaire. The specific scale was used as a continuous variable in the analysis (Zigmond \& Snaith, 1983).

\section{Statistical analysis}

All statistical analyses were performed using SPSS 23 (SPSS, Chicago, Illinois). Nominally significant alpha values were defined as $p<0.05$.

\section{Unadjusted linear regression analyses}

We used general linear models (GLM) with the previously described Sleep Index II first and then the sleep duration variable as the predictor, and the memory z-score as the outcome.

\section{Adjusted linear regression analyses} BMI.

Analyses were performed adjusting for: age, sex, education, and

As sleep medication can impact the cognitive performance of the participants, especially in the elderly (Otmani et al., 2008), we excluded from the initial sample those who were under sleep treatment. More specifically, the sleep medication variable included narcotics, hypnotics, antipsychotics, anticholinergics, and phenobarbital drugs.

In our initial analyses, we used the total sample, then, we excluded the demented participants, and lastly, we excluded both the demented and the MCI ones. We conducted further analyses only on the non-demented, non-MCI group adjusting also for the co-morbidities. We also conducted further analysis adding in the model of non-demented, non-MCI group anxiety and depression as covariates. Given the evidence showing association between $A p o E-\varepsilon 4$ and both sleep problems, as well as neurodegenerative diseases, we further stratified our sample by this specific gene.
ApoE- $\varepsilon 4$ genotypes were transformed into a dichotomous variable based on the number of $A p o E-\varepsilon 4$ alleles: 0 if the individual does not carry any copy of the $\varepsilon 4$ allele (non- $\varepsilon 4$ carriers) or 1 if the individual carries 1 or 2 copies of the $\varepsilon 4$ allele ( $\varepsilon 4$ carriers). Carriers of $\varepsilon 2 \varepsilon 4$ alleles were not included in the initial sample due to the opposite effect of these two alleles (Berge et al., 2014; Cacabelos, 2003; Corder \& Roses, 1996).

We further explored the interaction between gender and sleep quality/duration on memory performance. We also examined the associations between each individual element of the sleep questionnaire and memory. We then explored the existence of any other sleep disturbances in this group as described before. To further examine any association between sleep duration ranges and memory performance, we differentiated three sleep duration groups.

Guided by the existing literature, we further examined any difference between verbal and non-verbal memory. For more accuracy, we performed factor analysis which revealed that sum of total correct, immediate recall and delayed recall from GVLT loaded into the first factor (verbal memory), explaining the largest percentage of variance. Immediate and delayed recall from the Georgia test loaded into the second factor (non-verbal memory).

\section{Results}

The initial sample consisted of 1943 participants, all aged 65 years or older $(M=73, S D$ : 5.3$)$. After excluding participants being under sleep medication $(\mathrm{N}=184)$, and those with missing data, the sample included in our main analyses consisted of 1589 participants. The demographic characteristics of our sample are presented on Table 1.

From our non-demented, non-MCI group, 33 (4.8\%) participants reported sleep and nighttime behavior disorders, while $85(6.4 \%)$ reported having vivid dreams, and 28 (2.1\%) reported intense movements during sleep. 92 (7.4\%) participants met the RLS criteria.

In the total sample, longer sleep duration was related to low memory performance $(\mathrm{B}=-0.001, p \leq 0.0001)$, but not sleep quality and memory $(B=-0.038, p=0.121)$. After excluding the demented participants (final $\mathrm{N}=1520$ ), both the associations between sleep quality and memory $(B=-0.054, p=0.023)$ and sleep duration and memory ( $\mathrm{B}=-0.001, p \leq 0.0001$ ) were statistically significant. After excluding both the demented and the $\mathrm{MCl}$ participants (final $\mathrm{N}=1338$ ), associations between sleep quality

Table 1

Demographic and clinical characteristics of the study sample.

\begin{tabular}{|c|c|c|c|}
\hline Characteristics & $\begin{array}{l}\text { Total } \\
\text { sample }\end{array}$ & $\begin{array}{l}\text { Without } \\
\text { dementia }\end{array}$ & $\begin{array}{l}\text { Without } \\
\text { dementia-MCI }\end{array}$ \\
\hline Age, Mean years (SD) & $73(5.3)$ & $73(5.1)$ & $73(5.1)$ \\
\hline Gender, N (\% Female) & $\begin{array}{l}924 \\
(58.1)\end{array}$ & $892(59)$ & 789 (59) \\
\hline Education, Mean years (SD) & $7.7(4.8)$ & $7.7(4.7)$ & $7.8(4.7)$ \\
\hline BMI, Mean (SD) & $28.9(4.7)$ & $28.9(4.7)$ & $29(4.7)$ \\
\hline$\varepsilon 4$ carriers, $\mathrm{N}(\%)$ & $\begin{array}{l}184 \\
(17.6)\end{array}$ & $177(17.8)$ & $138(15)$ \\
\hline Anxiety, Mean (SD) & $\begin{array}{l}0.68 \\
(1.4)\end{array}$ & $0.69(1.4)$ & $0.64(1.4)$ \\
\hline Depression, yes (N (\%) & $\begin{array}{l}257 \\
(16.2)\end{array}$ & $239(15.7)$ & $192(14.4)$ \\
\hline $\begin{array}{l}\text { Sleep quality (averaged sum), } \\
\text { Mean (SD) }\end{array}$ & $\begin{array}{l}1.95 \\
(0.850)\end{array}$ & $1.96(0.848)$ & $1.95(0.852)$ \\
\hline $\begin{array}{l}\text { Sleep duration, Mean mins } \\
\quad \text { (SD) }\end{array}$ & $\begin{array}{l}390 \\
(91.4)\end{array}$ & 388 (88.9) & $388(87.4)$ \\
\hline Memory z-score, Mean (SD) & $\begin{array}{l}-0.2 \\
(0.9)\end{array}$ & $-0.1(0.9)$ & $-0.03(0.9)$ \\
\hline Total (N) & 1589 & 1499 & 1338 \\
\hline
\end{tabular}


and memory remained significant $(B=-0.065, p=0.006)$ (see Graph. 1), as did the associations between sleep duration and memory $(B=-0.001, p=0.003)$, with longer sleepers performing low in memory tests. After adding both sleep measures at the same regression model (excluding both the demented and the $\mathrm{MCI}$ participants), the associations remained significant for sleep quality and memory $(B=-0.072, p=0.003)$, as well as sleep duration and memory $(\mathrm{B}=-0.001, p \leq 0.0001)$. The results for the total sample, excluding the demented and excluding both the demented and the MCI participants are presented on Table 2.

Further analyses on the non-demented, non-MCI group: In our sample, the mean number of clinical co-morbidities was 2.1 $(\mathrm{SD}=1.5)$. After including the total number of clinical comorbidities in the model, the association between sleep quality and memory remained significant ( $\mathrm{B}=-0.068, p=0.005$ ), as well as the association between sleep duration and memory $(B=-0.001, p=0.003)$. The results did not change when we adjusted for the variable with the six most common comorbidities [sleep quality and memory $(B=-0.066, p=0.005)$ and sleep duration and memory performance $(\mathrm{B}=-0.001, p=0.003)]$.

Anxiety and depression: In the analysis of the non-demented, non-MCI group, after adding anxiety and depression in the model, the results remained significant for both the association between sleep quality and memory performance $(B=-0.066, p=0.006)$ and between sleep duration and memory as well $(B=-0.001$, $p=0.003)$. The association between the sleep duration groups [the short-sleepers $(\mathrm{N}=666,50 \%)$, the regular-sleepers $(\mathrm{N}=592$, $44.4 \%)$, and the long-sleepers $(\mathrm{N}=74,5.6 \%)$ ] and memory performance remained significant ( $B=-0.100, p=0.003$ ) (see Graph. 2).

Linear regression models revealed no significant interaction effect between sleep quality and gender on memory $(\mathrm{B}=-0.047$, $p=0.309$ ) or between sleep duration and gender on memory performance $(\mathrm{B}=0.000, p=0.913$ ).

From the 877 non-demented, non-MCI participants with data on the ApoE- $\varepsilon 4$ genotype, 154 (17.6\%) were classified as carriers of the gene. There was a significant association between sleep quality and memory $(\mathrm{B}=-0.082, p=0.017)$, as well as sleep dura- tion and memory $(\mathrm{B}=-0.001, p=0.006)$ for the non-carriers. We did not detect any significant association between sleep quality and memory $(B=-0.067, p=0.985)$ or sleep duration and memory ( $\mathrm{B}=-0.001, p=0.359$ ) for the $\varepsilon 4$-carriers.

Our analyses examining the relationships between memory and specific sleep questions, revealed significant associations for the following: time needed to fall asleep ( $\mathrm{B}=-0.033, p=0.025)$, the feeling that sleep was not quiet (moving restlessly, feeling tense, speaking etc., while sleeping) $(B=-0.034, p=0.010)$, getting enough sleep to feel rested in the morning $(B=-0.026, p=0.013)$ and the feeling of getting the amount of sleep needed $(\mathrm{B}=-0.034, p=0.001)$. The associations between memory and all the individual sleep questions are presented on Table 3. Even though this was an exploratory analysis, we further performed Bonferroni correction for the individual sleep questions. Results remained significant for the association between the question 'Get the amount of sleep needed' and memory performance.

Finally, in the adjusted model of non-demented, non-MCI participants, our analyses exploring the role of the two memory categories revealed that verbal memory was significantly associated with both sleep quality $(B=-0.202, p=0.009$ ), and sleep duration $(B=-0.002, p=0.016)$. The association remained significant for non-verbal memory and sleep duration $(B=-0.001, p=0.021$ ). There was no significant association, however, between nonverbal memory and sleep quality $(B=-0.027, p=630)$.

\section{Discussion}

In the current study, we examined the relationships between sleep quality as well as sleep duration and memory performance, in a large sample of older Greek adults. After controlling for age, sex, education, and BMI, poor sleep quality was associated with poor memory performance. The results remained significant even after adjusting for co-morbidities or anxiety and depression. Furthermore, participants who reported longer night-time sleep, had low memory scores. The two sex groups seemed to not behave differently on the associations between sleep quality and memory, as

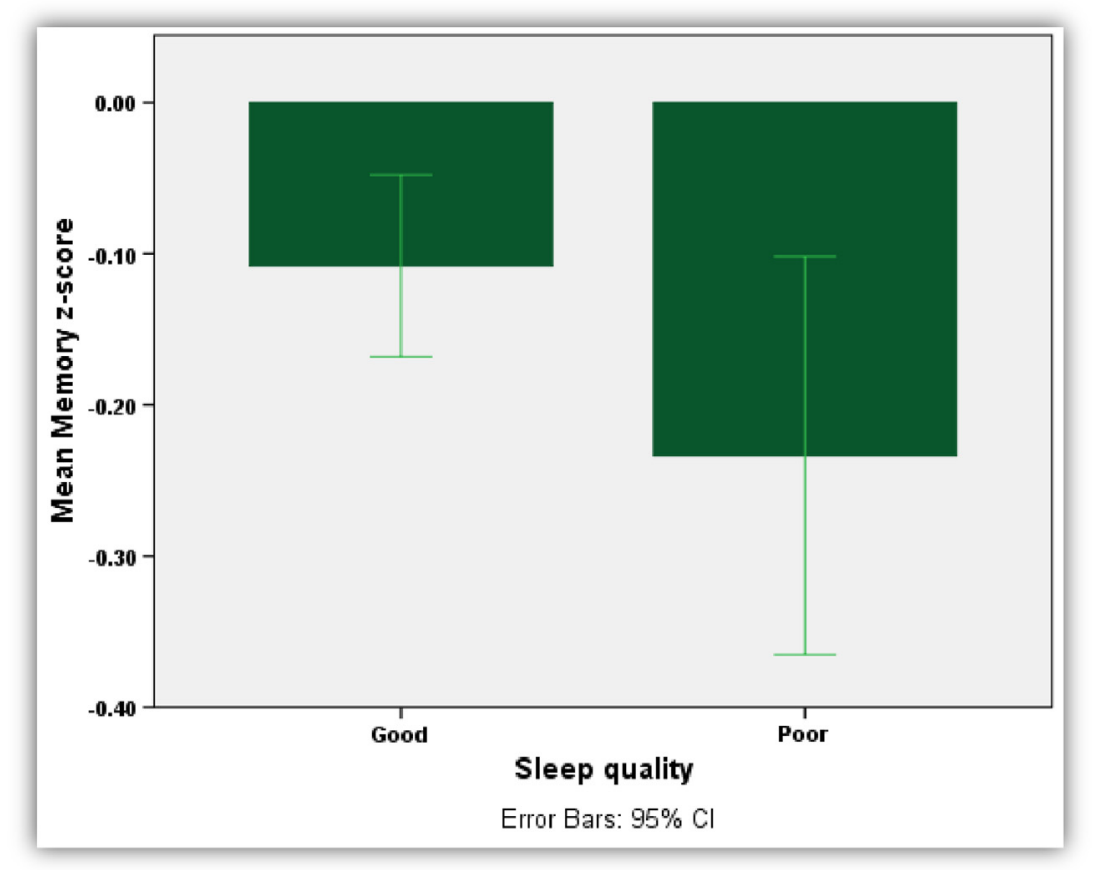

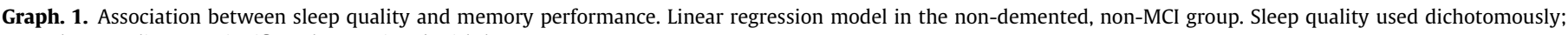
poor sleep quality was significantly associated with low memory z-scores. 
Table 2

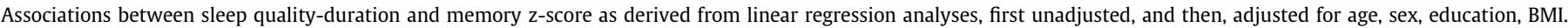

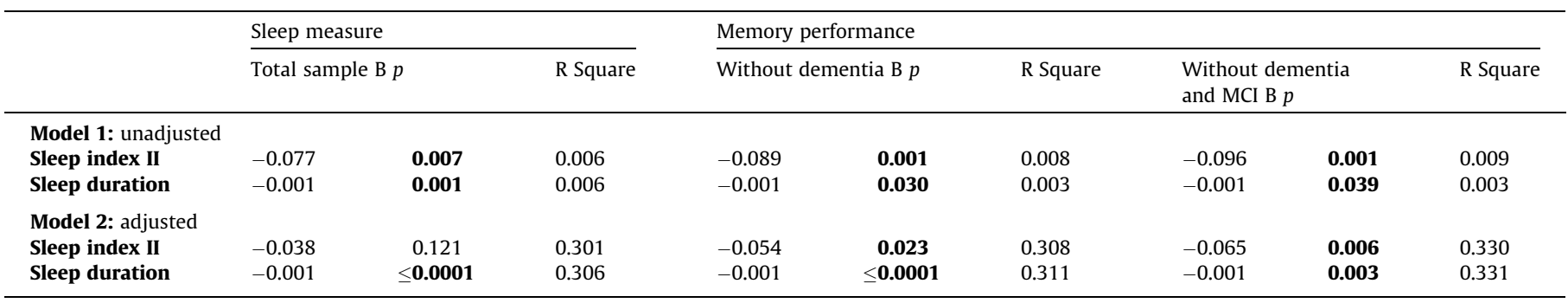

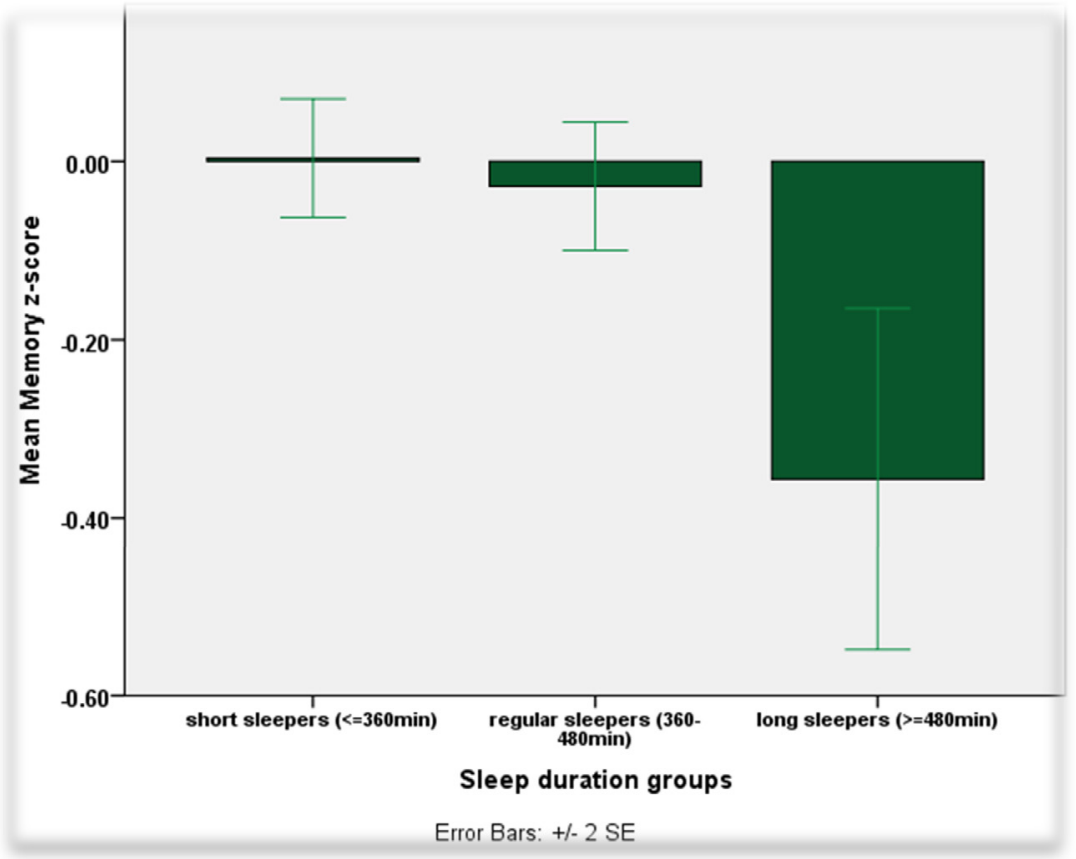

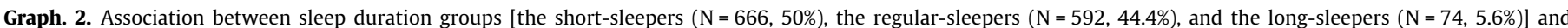
memory performance on the non-demented, non-MCI participants. Longer sleepers were significantly associated with low memory z-scores.

Table 3

Associations between individual sleep questions and memory. Linear regression models adjusted for age, sex, education, and BMI at the sample excluding demented and MCI participants. Unadjusted R squared (including only the covariates) $=0.326$.

\begin{tabular}{|c|c|c|c|}
\hline \multirow[t]{2}{*}{ Sleep question } & \multicolumn{3}{|c|}{ Memory performance } \\
\hline & $\mathrm{B}$ & $p$ & $\begin{array}{l}\text { Unadjusted R } \\
\text { Square }\end{array}$ \\
\hline Time to fall asleep & -0.033 & 0.025 & 0.328 \\
\hline Sleep not quiet & -0.034 & 0.010 & 0.331 \\
\hline Get enough sleep to feel rested & -0.026 & 0.013 & 0.330 \\
\hline Awake short of breath/headache & -0.029 & 0.182 & 0.327 \\
\hline Feel drowsy/sleepy during the day & -0.002 & 0.854 & 0.324 \\
\hline Have trouble falling asleep & 0.005 & 0.673 & 0.323 \\
\hline $\begin{array}{l}\text { Awake during sleep, trouble falling } \\
\text { asleep again }\end{array}$ & -0.015 & 0.260 & 0.325 \\
\hline Trouble staying awake during the day & -0.021 & 0.271 & 0.326 \\
\hline Snoring & 0.005 & 0.623 & 0.323 \\
\hline Napping during the day & -0.010 & 0.423 & 0.328 \\
\hline Get the amount of sleep needed & -0.034 & 0.001 & 0.332 \\
\hline
\end{tabular}

well as sleep duration and memory. The above associations were present to the non-carriers of the $\varepsilon 4$ allele of the ApoE gene. The most prominent sleep problems associated with low memory performance were: time to fall asleep, restless sleep, not enough sleep to feel rested, and report of not getting the amount of sleep needed.
Our findings indicate more memory deficits for the long sleepers and not the short ones. Even though most of the existing literature shows significant associations between both short and long sleep duration and low memory performance (Gildner et al., 2014; Kronholm et al., 2009; Lin et al., 2012), other studies provide mixed results regarding these associations (Faubel et al., 2009; Kawai et al., 2016; Miyata et al., 2013; Monaghan et al., 2016; Scullin, 2013). Based on our findings, long sleep duration could reflect both a greater physiological need for sleep, and/or a mechanism to compensate for poor sleep quality (Blackwell et al., 2006; Faubel et al., 2009).

The results revealed significant associations for sleep duration and both verbal and non-verbal memory. Sleep quality though was significantly linked to verbal memory only. Verbal memory has been linked to both sleep quality and sleep duration in existing studies (Clemens et al., 2005; Mu et al., 2005). The fewer studies examining the linkage between non-verbal memory and sleep, show mostly significant associations with sleep duration (Chee \& Chuah, 2007; Fernandez-Mendoza et al., 2010). This is in accordance to our findings. Interestingly, a different study suggests that obstructive sleep apnea, which is including both sleep quality and sleep duration problems, is associated with deficits in verbal but not non-verbal memory (Twigg et al., 2010). The different lateralization of cerebral activation in verbal and non-verbal memory 
could play a crucial role to the above associations (Breier et al., 1999; De Renzi \& Nichelli, 1975).

There are several possible biological explanations for our findings. Melatonin may play a moderating role in the association between sleep and memory. Melatonin is an hormone that anticipates the daily onset of darkness, acting as a sleep promoter and as previous evidence has shown, may prevent/slow the progression of neurodegenerative diseases (Hardeland, Pandi-Perumal, \& Cardinali, 2006). Furthermore, low melatonin levels have been related to poor hippocampal function, and specifically memory performance (Santoro et al., 2016). In a separate study, beneficial effects of drugs including melatonin were shown for memory enhancement in healthy and demented participants (Daulatzai, 2016). Thus, low levels of melatonin could explain both poor memory performance, and bad sleep quality in the older population.

Circadian rhythms functions could be another possible explanation accounting for the link between sleep and memory. Circadian rhythms are physical, mental and behavioral changes that follow a roughly 24-h cycle, responding primarily to light and darkness in an organism's environment. According to recent literature, circadian rhythms have been connected to hippocampal memory consolidation (Yoo \& Eckel-Mahan, 2016). Furthermore, as sleep duration has also been associated with circadian rhythms (Jiao et al., 2013), there might be a connection between sleep duration and poor memory performance.

In our attempt to define the link between sleep and memory, we cannot exclude the possibility that sleep dysregulation might be an initial symptom of an undetected subclinical neurodegenerative disease. According to existing longitudinal research, short and extended time in bed have been related to incident dementia in older adults (Bokenberger et al., 2016). Furthermore, memory performance has been predictive for incident dementia (Mowrey et al., 2016; Tomata et al., 2016). Amyloid- $\beta$ (A $\beta$ ) aggregation has been linked to $A D$ pathogenesis, and $A \beta$ concentrations have also been connected with sleep patterns (Lucey \& Bateman, 2014). Thus, both sleep dysregulations and bad memory performance could be indicative of an upcoming neurodegenerative disease. We tried to address this possibility by excluding not only the demented subjects, but even participants with MCI. Although the associations between sleep measures and memory were still present, the possibility of incipient neurodegeneration in nondemented, non-MCI subjects remains. Further longitudinal investigation is warranted in order to clarify the above associations.

ApoE- $\varepsilon 4$ has been linked to both sleep patterns (Gottlieb et al., 2004) and memory performance (Zlatar et al., 2016) in most of the existing literature. According to our findings, there were significant associations between sleep quality/duration and low memory performance in the non-carriers of the $\varepsilon 4$ allele. These results are not completely surprising as there is small but tangible literature showing no association between $A p o E-\varepsilon 4$ and sleep problems (Larkin et al., 2006; Saarelainen et al., 1998; Thakre et al., 2009; Tsapanou et al., 2015) or cognition (Turic et al., 2001). A possible explanation for these findings could be related to statistical power. As the effect size on the two groups is similar (sleep quality and memory: $\varepsilon 4$ carriers: $B=-0.08, \varepsilon 4$ non-carriers $B=-0.07$. Sleep duration and memory: $\varepsilon 4$ carriers: $\mathrm{B}=-0.001, \varepsilon 4$ non-carriers: $\mathrm{B}=-0.001$ ), statistical significance in the non $\varepsilon 4$ carriers may be related to the larger size of this group (compared to the smaller number of the $\varepsilon 4$ carriers). Another possible explanation could be of biological nature. Carriers of the $\varepsilon 4$ allele have been found to have greater Amyloid $\beta(\mathrm{A} \beta)$ burden than non-carriers (Strittmatter et al., 1993). Thus, sleep could relate to memory performance independently of the $A \beta$ pathway. If this is true, it could constitute additional evidence against the possibility of reverse causation in this cross-sectional study: if associations between sleep and cognition were the result of incipient neurodegenerative- $A \beta$ changes, one would expect that the associations would be stronger in $\varepsilon 4$ carriers that are known to harbor more of such pathologic burden. Another factor playing significant role in our results could be the subjectivity of our sleep questionnaire. A recent study interestingly showed no significant association between subjective sleep problems and ApoE-E4 carriers, in contrary to the association with objective sleep measures, indicating that objective sleep quality may proceed subjective sleep complaints in healthy older adults (Drogos et al., 2016). Future research would benefit from an examination of the influence of smallmodest genetic effects from multiple candidate genes in combination with specific environmental factors on sleep patterns.

In the present study there are some limitations that should be noted. First, we did not have the opportunity to use objective measures of sleep such as polysomnography or actigraphy. According to previous studies, self-reported sleep measures tend to provide an overestimation of sleep problems (Lauderdale et al., 2008; Van Den Berg et al., 2008). Moreover, the answers to the questionnaire referred to the last four weeks of the visiting time, and may not accurately represent chronic sleep patterns of the participants. Although we demonstrated convergent validity, full validation of the MOS scale in a Greek population is needed, ideally with objective sleep measures as the gold standard. Such work would be beneficial in establishing appropriate cut-off scores and norms for the Greek population for use in future research. In our analyses, effect sizes were small, which may partially stem from noise in sleep and cognitive instruments. The mixed results in the existing literature on the association between sleep and memory, and the low proportion of variability of memory performance explained by sleep measures may relate not only to sleep and cognition measurement issues. It is possible that many other parameters play an important role in this association. It is also conceivable that the true biological link between sleep and memory is feeble. The cross-sectional design of the study is another limitation, in so far as elucidating the directionality of the findings and further indicating the necessity of longitudinal analyses.

However, the current study has also several strengths. In our large sample of older adults, we used validated tests for the assessment of memory function, administered by trained psychologists or clinical neuropsychologists, in order to examine the association between both sleep quality, and sleep duration on memory performances in older adults. Furthermore, a clinical evaluation conducted by dementia experts in this study permitted a fine tuned classification of the study's sample cognitive status. We adjusted for many potential confounders. We performed secondarysupplementary analyses examining associations in participants at different cognitive strata. Our study also benefits from a unique sample, as it is drawn from a Mediterranean population. Given the potential cultural and sleep differences (i.e., siesta), as well as the lower proportions of $\varepsilon 4$ carriers (North-South European gradient) between these two samples, we considered it important to examine this topic in this specific sample. As the existing data in sleep and memory are not representative of this demographic, our sample may inform future research interested in crosscultural comparisons.

\section{Conclusions}

In summary, poor sleep quality and longer sleep duration were linked to low memory performance, in a group of older Greek adults. Further longitudinal research, as well as studies using objective sleep measures and biomarkers, are needed in order to shed more light into the probable biological underlying pathways of the above associations. Nevertheless, the present findings 
extend previous literature and may have significant public health implications for the older population.

\section{Acknowledgments}

This study was supported by the following grants: IIRG-09133014 from the Alzheimer's Association; 189 10276/8/9/2011 from the ESPA-EU program Excellence Grant (ARISTEIA), which is co-funded by the European Social Fund and Greek National resources, $\Delta Y 2 \beta /$ o1 $\kappa .51657 / 14.4 .2009$ from the Ministry for Health and Social Solidarity (Greece), 'Research excellence programs IKY/ Siemens' fellowship.

\section{Appendix A}

\section{Sleep Scale from the Medical Outcomes Study}

1. How long did it usually take for you to fall asleep during the past 4 weeks?

(Circle One)

$0-15 \mathrm{~min}$

$16-30 \mathrm{~min}$

1

$31-45 \mathrm{~min}$

2

46-60 min

2. On the average, how long did you sleep each night during the past 4 weeks?

Write in number of hours per night:

How often during the past 4 weeks did you:

3. Feel that your sleep was not quiet (moving restlessly, feeling tense, speaking, etc., while sleeping)?

4. Get enough sleep to feel rested upon waking in the morning?

5. Awake short of breath or with a headache?

6. Feel drowsy or sleepy during the day?

7. Have trouble falling asleep?

8. Awake during your sleep time and have trouble falling asleep again?

9. Have trouble staying awake during the day?

10. Snore during your sleep?

11. Take naps ( 5 min or longer) during the day?

12. Get the amount of sleep you needed?

Possible answers: 1 = All of the time, 2 = Most of the time, $3=\mathrm{A}$ good bit of the time, $4=$ Some of the time, $5=\mathrm{A}$ little of the time, $6=$ None of the time.

Hays, R. D., \& Stewart, A. L. (1992). Sleep measures. In A. L. Stewart \& J. E. Ware (Eds.). Measuring functioning and well-being: The Medical Outcomes Study approach (pp. 235-259), Durham, NC: Duke University Press.

Hays RD, Martin SA, Sesti AM, Spritzer KL. Psychometric properties of the Medical Outcomes Study Sleep measure. Sleep Med. 2005;6(1):41-4.

\section{References}

Allen, R. P. et al. (2003). Restless legs syndrome: diagnostic criteria, special considerations, and epidemiology. A report from the restless legs syndrome diagnosis and epidemiology workshop at the National Institutes of Health. Sleep Medicine, 4(2), 101-119.

Aly, M., \& Moscovitch, M. (2010). The effects of sleep on episodic memory in older and younger adults. Memory, 18(3), 327-334.

Armitage, R. et al. (1997). Relationship between objective and subjective sleep measures in depressed patients and healthy controls. Depress Anxiety, 5(2), 97-102.

Backhaus, J. et al. (2007). Midlife decline in declarative memory consolidation is correlated with a decline in slow wave sleep. Learning E Memory, 14(5), 336-341.

Basner, M. et al. (2007). American time use survey: Sleep time and its relationship to waking activities. Sleep, 30(9), 1085-1095.
Berge, G. et al. (2014). Apolipoprotein E epsilon2 genotype delays onset of dementia with Lewy bodies in a Norwegian cohort. Journal of Neurology, Neurosurgery and Psychiatry, 85(11), 1227-1231.

Blackwell, T. et al. (2006). Poor sleep is associated with impaired cognitive function in older women: The study of osteoporotic fractures. Journals of Gerontology. Series A, Biological Sciences and Medical Sciences, 61(4), 405-410.

Blessed, G., Tomlinson, B. E., \& Roth, M. (1968). The association between quantitative measures of dementia and of senile change in the cerebral grey matter of elderly subjects. British Journal of Psychiatry, 114(512), 797-811.

Bokenberger, K. et al. (2016). Association between Sleep characteristics and incident dementia accounting for baseline cognitive status: A prospective populationbased study. Journals of Gerontology. Series A, Biological Sciences and Medical Sciences.

Breier, J. I. et al. (1999). Lateralization of cerebral activation in auditory verbal and non-verbal memory tasks using magnetoencephalography. Brain Topography, 12 (2), 89-97.

Cacabelos, R. (2003). The application of functional genomics to Alzheimer's disease. Pharmacogenomics, 4(5), 597-621.

Chee, M. W., \& Chuah, Y. M. (2007). Functional neuroimaging and behavioral correlates of capacity decline in visual short-term memory after sleep deprivation. Proceedings of the National Academy of Sciences USA, 104(22), 9487-9492.

Clemens, Z., Fabo, D., \& Halasz, P. (2005). Overnight verbal memory retention correlates with the number of sleep spindles. Neuroscience, 132(2), 529-535.

Corder, E. H., \& Roses, A. D. (1996). No increased risk of the apolipoprotein E epsilon2 allele with early-onset Alzheimer's disease. Annals of Neurology, 39(3), 414-416.

Cummings, J. L. et al. (1994). The Neuropsychiatric Inventory: Comprehensive assessment of psychopathology in dementia. Neurology, 44(12), 2308-2314.

Dardiotis, E. et al. (2014). The Hellenic Longitudinal Investigation of Aging and Diet (HELIAD): Rationale, study design, and cohort description. Neuroepidemiology, 43(1), 9-14.

Daulatzai, M. A. (2016). Pharmacotherpy and Alzheimer's Disease: The M-Drugs (Melatonin, Minocycline, Modafinil, and Memantine) approach. Current Pharmaceutical Design, 22(16), 2411-2430.

De Renzi, E., \& Nichelli, P. (1975). Verbal and non-verbal short-term memory impairment following hemispheric damage. Cortex, 11(4), 341-354.

Drogos, L. L. et al. (2016). Evidence of association between sleep quality and APOE epsilon4 in healthy older adults: A pilot study. Neurology, 87(17), 1836-1842.

Faubel, R. et al. (2009). Usual sleep duration and cognitive function in older adults in Spain. Journal of Sleep Research, 18(4), 427-435.

Fernandez-Mendoza, J. et al. (2010). Insomnia with objective short sleep duration is associated with deficits in neuropsychological performance: A general population study. Sleep, 33(4), 459-465.

Fountoulakis, K.N., Tsolaki, M., Iacovides, A., Yesavage, J., O'Hara, R., Kazis, A., Ierodiakonou, C. (1999). The validation of the short form of the Geriatric Depression Scale (GDS) in Greece. Aging (Milano) 11(6), 367-72.

Garcia-Borreguero, D. et al. (2006). Epidemiology of restless legs syndrome: The current status. Sleep Medicine Reviews, 10(3), 153-167.

Gildner, T. E. et al. (2014). Associations between sleep duration, sleep quality, and cognitive test performance among older adults from six middle income countries: Results from the Study on Global Ageing and Adult Health (SAGE). Journal of Clinical Sleep Medicine, 10(6), 613-621.

Gottlieb, D. J. et al. (2004). APOE epsilon4 is associated with obstructive sleep apnea/hypopnea: The Sleep Heart Health Study. Neurology, 63(4), 664-668.

Grewe, P. et al. (2016). Subjective memory complaints in patients with epilepsy: The role of depression, psychological distress, and attentional functions. Epilepsy Research, 127, 78-86.

Hardeland, R., Pandi-Perumal, S. R., \& Cardinali, D. P. (2006). Melatonin. International Journal of Biochemistry \& Cell Biology, 38(3), 313-316.

Hays, R.D., Sherbourne, C. D., Mazel, R.M. (1995), User's Manual for the Medical Outcomed Study (MOS) Core Measures of Health-Related Quality of Life (Published by RAND).

Hita-Yanez, E., Atienza, M., \& Cantero, J. L. (2013). Polysomnographic and subjective sleep markers of mild cognitive impairment. Sleep, 36(9), 1327-1334.

Jiao, L. et al. (2013). Sleep duration and incidence of colorectal cancer in postmenopausal women. British Journal of Cancer, 108(1), 213-221.

Jorm, A. F. (2000). Is depression a risk factor for dementia or cognitive decline? A review. Gerontology, 46(4), 219-227.

Kavouras, S.A., Maraki, M., Kollia, M., Gioxari, A., Jansen, L.T., Sidossis, L.S. (2016). Development, reliability and validity of a physical activity questionnaire for estimating energy expenditure in Greek adults. Science and Sports 31, e47-e53

Kawai, M. et al. (2016). Delta activity at sleep onset and cognitive performance in community-dwelling older adults. Sleep, 39(4), 907-914.

Kronholm, E. et al. (2009). Self-reported sleep duration and cognitive functioning in the general population. Journal of Sleep Research, 18(4), 436-446.

Landis, C. A. et al. (2003). Self-reported sleep quality and fatigue correlates with actigraphy in midlife women with fibromyalgia. Nursing Research, 52(3), $140-147$.

Larkin, E. K. et al. (2006). Apolipoprotein E and obstructive sleep apnea: Evaluating whether a candidate gene explains a linkage peak. Genetic Epidemiology, 30(2), $101-110$.

Lauderdale, D. S. et al. (2008). Self-reported and measured sleep duration: How similar are they? Epidemiology, 19(6), 838-845.

Lawton, M. P., \& Brody, E. M. (1969). Assessment of older people: Self-maintaining and instrumental activities of daily living. Gerontologist, 9(3), 179-186. 
Lin, P. C. et al. (2012). Atypical work schedules are associated with poor sleep quality and mental health in Taiwan female nurses. International Archives of Occupational and Environmental Health, 85(8), 877-884.

Lucey, B. P., \& Bateman, R. J. (2014). Amyloid-beta diurnal pattern: Possible role of sleep in Alzheimer's disease pathogenesis. Neurobiology of Aging, 35(Suppl 2), S29-e34.

Meador, K. J. et al. (1993). The role of cholinergic systems in visuospatial processing and memory. Journal of Clinical and Experimental Neuropsychology, 15(5), $832-842$.

Miller, M. A. et al. (2014). Cross-sectional study of sleep quantity and quality and amnestic and non-amnestic cognitive function in an ageing population: The English Longitudinal Study of Ageing (ELSA). PLoS ONE, 9(6), e100991.

Miyata, S. et al. (2013). Poor sleep quality impairs cognitive performance in older adults. Journal of Sleep Research, 22(5), 535-541.

Monaghan, P. et al. (2016). Hemispheric processing of memory is affected by sleep. Brain and Language.

Mowrey, W. B. et al. (2016). Memory binding test predicts incident amnestic mild cognitive impairment. Journal of Alzheimer's Disease, 53(4), 1585-1595.

$\mathrm{Mu}$, O. et al. (2005). Decreased cortical response to verbal working memory following sleep deprivation. Sleep, 28(1), 55-67.

Nebes, R. D. et al. (2009). Self-reported sleep quality predicts poor cognitive performance in healthy older adults. Journals of Gerontology. Series B, Psychological Sciences and Social Sciences, 64(2), 180-187.

Ohayon, M. M. (2004). Sleep and the elderly. Journal of Psychosomatic Research, 56 (5), 463-464.

Ohayon, M. M. et al. (2004). Meta-analysis of quantitative sleep parameters from childhood to old age in healthy individuals: Developing normative sleep values across the human lifespan. Sleep, 27(7), 1255-1273.

Otmani, S. et al. (2008). Effects of prolonged-release melatonin, zolpidem, and thei combination on psychomotor functions, memory recall, and driving skills in healthy middle aged and elderly volunteers. Human Psychopharmacology, 23(8), 693-705.

Ramos, A. R. et al. (2013). Association between sleep duration and the mini-menta score: The Northern Manhattan study. Journal of Clinical Sleep Medicine, 9(7), 669-673.

Riegel, B., \& Weaver, T. E. (2009). Poor sleep and impaired self-care: Towards comprehensive model linking sleep, cognition, and heart failure outcomes. European Journal of Cardiovascular Nursing, 8(5), 337-344.

Saarelainen, S. et al. (1998). No relation between apolipoprotein E alleles and obstructive sleep apnea. Clinical Genetics, 53(2), 147-148.

Santoro, S. D. et al. (2016). Correlations between behavior, memory, sleep-wake an melatonin in Williams-Beuren syndrome. Physiology \& Behavior, 159, 14-19.

Schmutte, T. et al. (2007). The relation between cognitive functioning and selfreported sleep complaints in nondemented older adults: Results from the Bronx aging study. Behavioral Sleep Medicine, 5(1), 39-56.

Scullin, M. K. (2013). Sleep, memory, and aging: the link between slow-wave sleep and episodic memory changes from younger to older adults. Psychology and Aging, 28(1), 105-114.

Scullin, M. K., \& Bliwise, D. L. (2015). Sleep, cognition, and normal aging: Integrating a half century of multidisciplinary research. Perspectives on Psychological Science, 10(1), 97-137.
Spritzer, K.L., Hays, R.D. (2003). MOS Sleep Scale: A manual for use and scoring, Version 1.0. Los Angeles, CA

Strittmatter, W. J. et al. (1993). Apolipoprotein E: High-avidity binding to betaamyloid and increased frequency of type 4 allele in late-onset familial Alzheimer disease. Proceedings of the National Academy of Sciences USA, 90(5), 1977-1981.

Thakre, T. P., Mamtani, M. R., \& Kulkarni, H. (2009). Lack of association of the APOE epsilon 4 allele with the risk of obstructive sleep apnea: Meta-analysis and meta-regression. Sleep, 32(11), 1507-1511.

Tomata, Y., et al. (2016). Predictive ability of a simple subjective memory complaints scale for incident dementia: Evaluation of Japan's national checklist, the "Kihon Checklist". Geriatrics \& Gerontology International.

Tsapanou, A. et al. (2015). Examining the association between Apolipoprotein E (APOE) and self-reported sleep disturbances in non-demented older adults. Neuroscience Letters, 606, 72-76.

Turic, D. et al. (2001). No association between apolipoprotein E polymorphisms and general cognitive ability in children. Neuroscience Letters, 299(1-2), 97-100.

Twigg, G. L. et al. (2010). Obstructive sleep apnea syndrome is associated with deficits in verbal but not visual memory. American Journal of Respiratory and Critical Care Medicine, 182(1), 98-103.

Van Den Berg, J. F. et al. (2008). Disagreement between subjective and actigraphic measures of sleep duration in a population-based study of elderly persons. Journal of Sleep Research, 17(3), 295-302.

Vlahou, C. H. et al. (2013). Development of the Greek Verbal Learning Test: Reliability, construct validity, and normative standards. Archives of Clinical Neuropsychology, 28(1), 52-64.

Walker, M. P. et al. (2000). The Clinician Assessment of Fluctuation and the One Day Fluctuation Assessment Scale. Two methods to assess fluctuating confusion in dementia. British Journal of Psychiatry, 177, 252-256.

Xu, L. et al. (2011). Short or long sleep duration is associated with memory impairment in older Chinese: The Guangzhou Biobank Cohort Study. Sleep, 34 (5), 575-580.

Xu, L. et al. (2014). Sleep duration and memory in the elderly Chinese: Longitudinal analysis of the Guangzhou Biobank Cohort Study. Sleep, 37(11), 1737-1744.

Yaffe, K. et al. (2011). Sleep-disordered breathing, hypoxia, and risk of mild cognitive impairment and dementia in older women. JAMA, 306(6), 613-619.

Yesavage, J. A. (1988). Geriatric depression scale. Psychopharmacology Bulletin, 24(4), 709-711.

Yoo, S. H., \& Eckel-Mahan, K. (2016). Hippocampal PER1: A circadian sentinel controlling RSKy activity during memory formation. Journal of Neurochemistry, 138(5), 650-652.

Young, T., Skatrud, J., \& Peppard, P. E. (2004). Risk factors for obstructive sleep apnea in adults. JAMA, 291(16), 2013-2016.

Zigmond, A. S., \& Snaith, R. P. (1983). The hospital anxiety and depression scale. Acta Psychiatrica Scand., 67(6), 361-370.

Zlatar, M. et al. (2016). Origin of the zero-field splitting in mononuclear octahedral Mn(IV) complexes: A combined experimental and theoretical investigation. Inorganic Chemistry, 55(3), 1192-1201. 